\title{
Article
}

\section{Thermoelectric Properties of Two-Dimensional Molybdenum-based MXenes}

\author{
Hyunho Kim, Babak Anasori, Yury Gogotsi, and Husam N. Alshareef
}

Chem. Mater., Just Accepted Manuscript • DOI: 10.1021/acs.chemmater.7b02056 • Publication Date (Web): 05 Jul 2017

Downloaded from http://pubs.acs.org on July 12, 2017

\section{Just Accepted}

"Just Accepted" manuscripts have been peer-reviewed and accepted for publication. They are posted online prior to technical editing, formatting for publication and author proofing. The American Chemical Society provides "Just Accepted" as a free service to the research community to expedite the dissemination of scientific material as soon as possible after acceptance. "Just Accepted" manuscripts appear in full in PDF format accompanied by an HTML abstract. "Just Accepted" manuscripts have been fully peer reviewed, but should not be considered the official version of record. They are accessible to all readers and citable by the Digital Object Identifier (DOI®). "Just Accepted" is an optional service offered to authors. Therefore, the "Just Accepted" Web site may not include all articles that will be published in the journal. After a manuscript is technically edited and formatted, it will be removed from the "Just Accepted" Web site and published as an ASAP article. Note that technical editing may introduce minor changes to the manuscript text and/or graphics which could affect content, and all legal disclaimers and ethical guidelines that apply to the journal pertain. ACS cannot be held responsible for errors or consequences arising from the use of information contained in these "Just Accepted" manuscripts. 


\section{INTRODUCTION}

A large class of two-dimensional (2D) transition metal carbides and nitrides, also known as MXenes, has been under extensive investigation since its first discovery in 2011. ${ }^{1}$ These atomically thin materials, with general formula of $M_{n+1} X_{n} T_{x}$, are usually obtained by selective removal of the 'A-element' (Al, Ga, etc.) layers from their ternary layered $M_{n+1} A X_{n}$ phase and similar layered precursors. In MXenes, $\mathrm{M}$ is an early transition metal, $X$ is carbon and/or nitrogen, $T_{x}$ represents the surface functional groups such as $\mathrm{OH}, \mathrm{O}$, and/or $\mathrm{F}$ groups, and $\mathrm{n}=1,2$, or 3 . Twenty different MXenes ${ }^{2,3}$ have been experimentally synthesized for applications ranging from energy storage, ${ }^{4-10}$ to electromagnetic interference shielding, ${ }^{11}$ biosensing, ${ }^{12-14}$ ion sieving, ${ }^{15}$ water purification, ${ }^{16}$ and antibacterial activity. ${ }^{17}$ As there are more than 70 different MAX phases that have been experimentally prepared, many more MXenes are expected to be explored. ${ }^{18}$

Theoretical studies have predicted that most MXenes are metallic, but few of them $\left(\mathrm{Sc}_{2} \mathrm{CT}_{\mathrm{x}}, \mathrm{Ti}_{2} \mathrm{CT}_{\mathrm{x}}, \mathrm{Zr}_{2} \mathrm{CT}_{\mathrm{x}}\right.$, and $\left.\mathrm{Hf}_{2} \mathrm{CT}_{\mathrm{x}}\right)$ are predicted to have a non-zero band gap when terminated with appropriate surface groups. ${ }^{19-21}$ These semiconducting MXenes are expected to have a high Seebeck coefficient at low temperatures; e.g. $\sim 1140 \mu \mathrm{V} \mathrm{K}^{-1}$ and $\sim 2200 \mu \mathrm{V} \mathrm{K}^{-1}$ at 100 $\mathrm{K}$ predicted for $\mathrm{Ti}_{2} \mathrm{CO}_{2}$ and $\mathrm{Sc}_{2} \mathrm{C}(\mathrm{OH})_{2}$, respectively. According to a later calculation study, $\mathrm{Mo}_{2} \mathrm{CF}_{2}$ (with fluorineterminated surface) has been reported to have superior thermoelectric power factor among the other 35 different functionalized $\mathrm{M}_{2} \mathrm{XT}_{\mathrm{x}}$ MXene systems. ${ }^{22}$ However, those theoreti- cal studies have assumed a fully controlled surface termination, which may be challenging to achieve in experimental studies, where multiple functional groups co-exist. Hope et al reported that the portion of the surface termination groups of $\mathrm{Ti}_{3} \mathrm{C}_{2} \mathrm{~T}_{\mathrm{x}}$ MXene is dependent on the synthesis routes, and experimentally quantified by ${ }^{1} \mathrm{H}$ and ${ }^{19} \mathrm{~F}$ nuclear magnetic resonance spectroscopy, ${ }^{23}$ where HF etching route results in higher proportion of fluorine termination.

In this work, we report the thermoelectric properties of three kinds of Mo-based MXenes - $\mathrm{M}_{2} \mathrm{CT}_{\mathrm{x}}, \mathrm{M}_{3} \mathrm{C}_{2} \mathrm{~T}_{\mathrm{x}}$, and $\mathrm{M}_{4} \mathrm{C}_{3} \mathrm{~T}_{\mathrm{x}}$. Freestanding MXene papers were fabricated by vacuumassisted filtration of suspensions of delaminated $2 \mathrm{D} \mathrm{Mo}_{2} \mathrm{CT}_{\mathrm{x}}$, $\mathrm{Mo}_{2} \mathrm{TiC}_{2} \mathrm{~T}_{\mathrm{x}}$, and $\mathrm{Mo}_{2} \mathrm{Ti}_{2} \mathrm{C}_{3} \mathrm{~T}_{\mathrm{x}}$. The latter two compositions were selected instead of $\mathrm{Mo}_{3} \mathrm{C}_{2} \mathrm{~T}_{\mathrm{x}}$ and $\mathrm{Mo}_{4} \mathrm{C}_{3} \mathrm{~T}_{\mathrm{x}}$, which are not thermodynamically stable and thus difficult to synthesize. ${ }^{24,25}$ $\mathrm{Mo}_{2} \mathrm{TiC}_{2} \mathrm{~T}_{\mathrm{x}}$, and $\mathrm{Mo}_{2} \mathrm{Ti}_{2} \mathrm{C}_{3} \mathrm{~T}_{\mathrm{x}}$ belong to the family of ordered double-transition-metal carbide MXenes, ${ }^{26}$ in which Mo atoms are preferentially located in the outer layers, sandwiching the layers of Ti. As a result, all three Mo-based MXenes that we synthesized in this study have Mo atoms on the surface, creating a similar surface chemistry. Additionally, these Mo-based MXenes with oxygen termination have been reported as 2D topological insulators, based on calculation along with the consideration of spin-orbit coupling. ${ }^{27-29} 2 \mathrm{D}$ topological insulators with controlled size have been predicted to show anomalous Seebeck effects, ${ }^{30}$ and the best thermoelectric material, $\mathrm{Bi}_{2} \mathrm{Te}_{3}$, and its alloys are also topological insulators. ${ }^{31}$ This provided additional motivation for studying transport properties of these MXenes. 

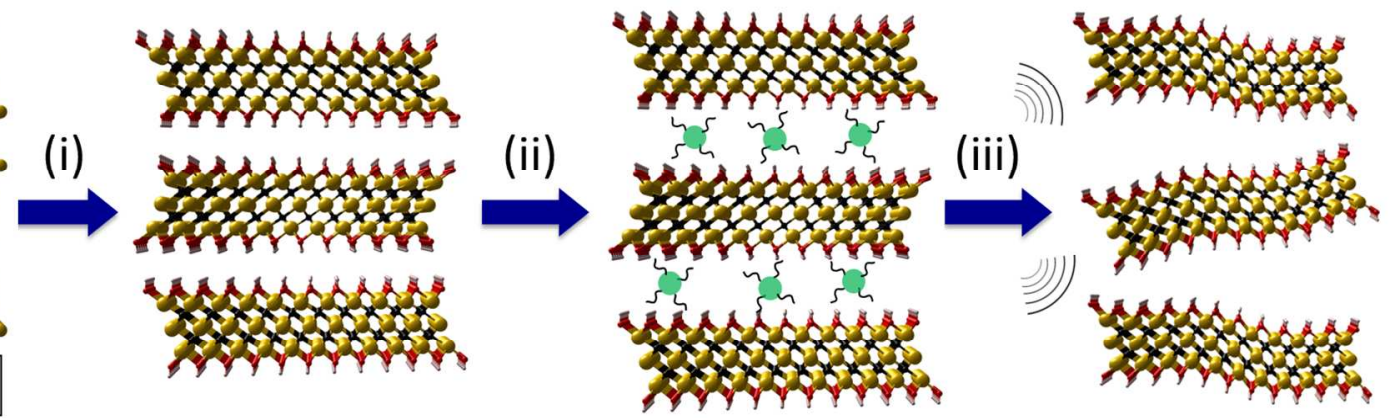

Figure 1. Schematic illustration of MXene synthesis process. (i) selective etching of the A-element in the layered precursor materials, (ii) intercalation of organic molecules, tetrabutylammonium cations $\left(\mathrm{TBA}^{+}\right)$, (iii) exfoliation of 2D MXene flakes by molecule exchange with water and ultra-sonication.

\section{Experimental Methods}

Synthesis of MXene freestanding papers. To synthesize $\mathrm{Mo}_{2} \mathrm{CT}_{\mathrm{x}},{ }^{32} \mathrm{Mo}_{2} \mathrm{TiC}_{2} \mathrm{~T}_{\mathrm{x}},{ }^{26}$ and $\mathrm{Mo}_{2} \mathrm{Ti}_{2} \mathrm{C}_{3} \mathrm{~T}_{\mathrm{x}}{ }^{26}$ MXenes, $2 \mathrm{~g}$ of $\mathrm{Mo}_{2} \mathrm{Ga}_{2} \mathrm{C}$ powder was slowly added into $80 \mathrm{ml}$ of $25 \%$ aqueous hydrofluoric acid (HF), and for $\mathrm{Mo}_{2} \mathrm{TiAlC}_{2}$ and $\mathrm{Mo}_{2} \mathrm{Ti}_{2} \mathrm{AlC}_{3}$ MAX phases $20 \mathrm{ml}$ of $50 \% \mathrm{HF}$ were used. The precursor carbides were slowly added into the acidic solutions which were cooled by ice-bath to minimize the localized heating. The mixtures were then stirred with a magnetic Teflon stir bar at $55^{\circ} \mathrm{C}$ for $160 \mathrm{~h}, 48 \mathrm{~h}$, and $96 \mathrm{~h}$, respectively. After etching, the mixtures were washed several times until the $\mathrm{pH}$ reached around 6. For each washing step, $40 \mathrm{ml}$ of deionized (DI) water was added into centrifuge tubes, hand shaken for 1 min, centrifuged at $5000 \mathrm{rpm}$ for $2 \mathrm{~min}$, and then discarded the supernatant. The solid sediments were kept in a vacuum oven at RT overnight for drying. 1g of dried multilayer MXene powder was further re-dispersed into $10 \mathrm{ml}$ of $54-56 \%$ tetrabutylammonium hydroxide (TBAOH) solution for intercalation, and stirred for $4 \mathrm{~h} \quad\left(\mathrm{Mo}_{2} \mathrm{CT}_{\mathrm{x}}, \mathrm{Mo}_{2} \mathrm{TiC}_{2} \mathrm{~T}_{\mathrm{x}}\right)$ or $18 \mathrm{~h}$ $\left(\mathrm{Mo}_{2} \mathrm{Ti}_{2} \mathrm{C}_{3} \mathrm{~T}_{\mathrm{x}}\right)$ at $\mathrm{RT}$. The resulting mixtures were washed two times, and sediments were re-dispersed into DI water followed by ultra-sonication for an hour. The mixtures were later centrifuged at $5000 \mathrm{rpm}$ for 1 hour, and the resulting supernatants were carefully collected. The suspension solutions were vacuum filtrated through a porous membrane (3501, surfactantcoated PP, Celgard, USA), and the vacuum filtrated papers were carefully peeled off for the experiment. 50\% HF and 54$56 \% \mathrm{TBAOH}$ were purchased from Aldrich.

Thermoelectric properties measurement. Electrical conductivity and Seebeck coefficient of MXene paper were measured in the in-plane direction under dynamic flow of $\mathrm{Ar} / \mathrm{H}_{2}$ reducing ambient. The measurements were done in the temperature range of 300-800 K, using a commercially available thermoelectric tester RZ2001i (Ozawa Science Co. Ltd., Japan). At each temperature, electrical conductivity were measured under thermal equilibrium by 4-point linear probe method. After that, a small thermal gradient was generated by local cooling of one side of sample (local cooling was done by air circulation through one of the quartz sample holder pipe). Among the four linear probes, two of the inner probes are thermocouple-probes, so the temperature difference $(\Delta \mathrm{T})$ and the voltage difference $(\Delta \mathrm{V})$ can be measured across the sample. Five different data points were collected at each temperature, and the Seebeck coefficient could be calculated by finding the linear fit slope
$(\Delta \mathrm{V} / \Delta \mathrm{T})$. As the measured Seebeck coefficient is the difference between the sample and the platinum electrode $\left(S_{\text {measured }}\right.$ $=\mathrm{S}_{\text {sample }}-\mathrm{S}_{\text {electrode }}$ ), corresponding Seebeck coefficient of Pt at the temperature was accounted for to find the Seebeck coefficient of the sample. Thermal history for the measurement is shown in Figure S6. Further details on the measurement can be found elsewhere. ${ }^{33}$

Characterization. X-ray diffraction (XRD) patterns were collected by a Bruker diffractometer (D8 advance, AXS system, Germany) with $\mathrm{Cu} \mathrm{K} \alpha$ radiation, $\lambda=1.5406 \AA$. For the precursor carbides, small amounts of powder were loaded into a small cavity of the sample holder and pressed using a glass slide. For MXene, a single slice of MXene paper was placed on a zero-diffraction plate. Cross-section morphologies were observed by FE-SEM (Nova Nano 630, FEI, USA). The Raman spectra were collected by a micro-Raman spectrometer (LabRAM Aramis, Horiba, Japan) equipped with a long working distance $\times 50$ objective lens (LMPLFLN50X, Olympus, Japan) using $473 \mathrm{~nm}$ excitation (04-01 series, Cobalt blues ${ }^{\mathrm{TM}}$, Sweden) at RT. Thermal analysis (TGA-DSC) was carried out by STA 449 F1 Jupiter (Netzsch, Germany) with the heating rate of $5 \mathrm{~K} \mathrm{~min}^{-1}$ under $\mathrm{Ar}$ ambient, using $\mathrm{Al}_{2} \mathrm{O}_{3}$ crucibles. Carrier concentration and Hall mobility were measured by a Hall-effect measurement system (Lake Shore 7700A) using van der Pauw technique at RT under a magnetic field range of $\pm 5,10,15$, and $20 \mathrm{kG}$. Ohmic contact was achieved by using fast drying silver paste.

\section{RESULT AND DISCUSSION}

Figure 1 shows a schematic illustration of the MXene synthesis process. The ternary and quaternary carbide precursor powders $\left(\mathrm{Mo}_{2} \mathrm{Ga}_{2} \mathrm{C},{ }^{34} \mathrm{Mo}_{2} \mathrm{TiAlC}_{2},{ }^{35}\right.$ and $\mathrm{Mo}_{2} \mathrm{Ti}_{2} \mathrm{AlC}_{3}{ }_{3}$ ) were first chemically etched by hydrofluoric acid (HF) which attacks the metallic M-A bond. ${ }^{2}$ After washing in deionized water several times, these multilayer MXenes (HF-etched powders) were further reacted with tetrabutylammonium hydroxide (TBAOH) which leads to exfoliation of 2D MXene suspensions by intercalation of tetrabutylammonium cation $\left(\mathrm{TBA}^{+}\right)$between the negatively charged MXene sheets, followed by further washing and sonication. Details of the synthesis process are explained in the experimental section. Scanning electron microscopy (SEM) images of the precursors and multilayer MXenes (Figure S1) show the typical layered 

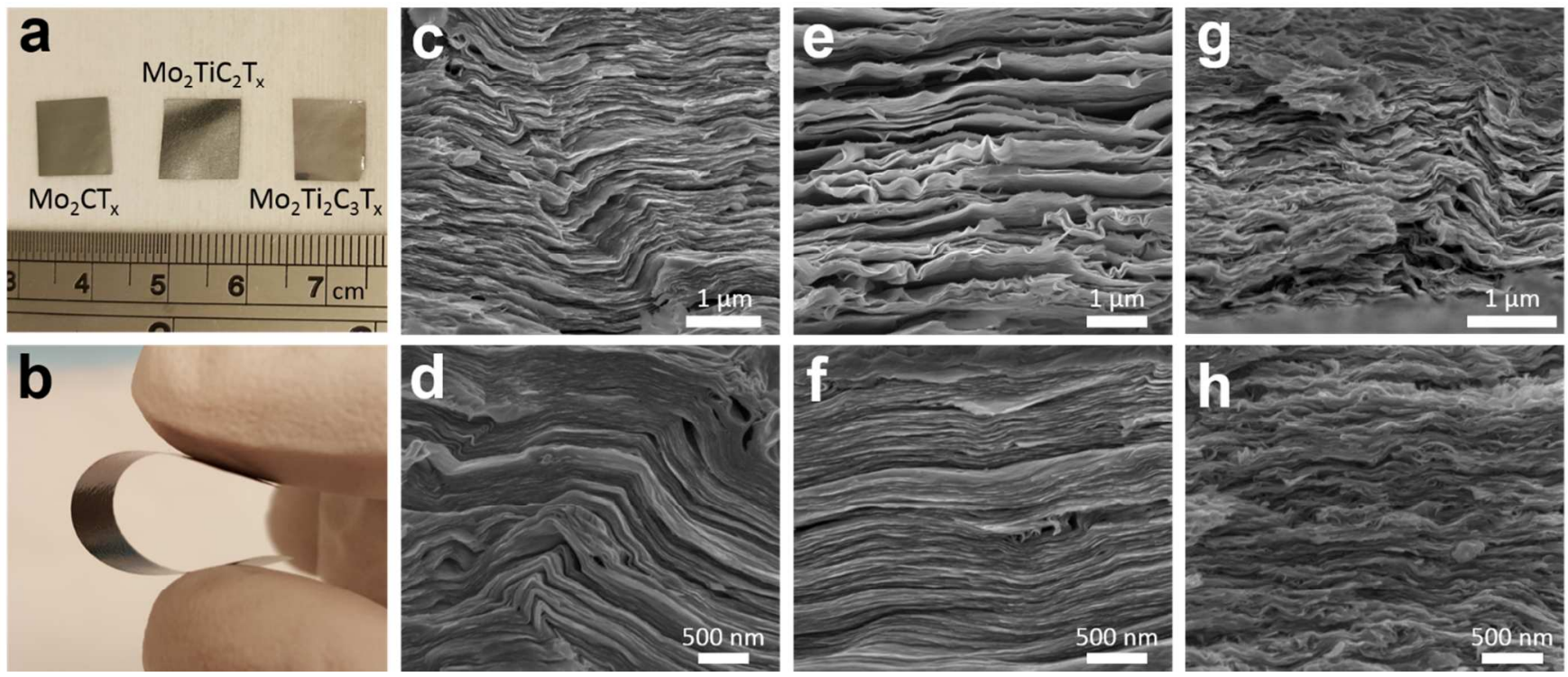

Figure 2. (a) Digital photographs of Mo-based MXene papers. (b) Demonstration of the flexibility of freestanding $\mathrm{Mo}_{2} \mathrm{Ti}_{2} \mathrm{C}_{3} \mathrm{~T}_{\mathrm{x}}$ paper in pristine state. (c-h) Cross-section SEM micrographs of Mo-based MXene papers; (c) pristine $\mathrm{Mo}_{2} \mathrm{CT}_{\mathrm{x}},(\mathrm{d})$ annealed $\mathrm{Mo}_{2} \mathrm{CT}_{\mathrm{x}}$, (e) pristine $\mathrm{Mo}_{2} \mathrm{TiC}_{2} \mathrm{~T}_{\mathrm{x}}$, (f) annealed $\mathrm{Mo}_{2} \mathrm{TiC}_{2} \mathrm{~T}_{\mathrm{x}},(\mathrm{g})$ pristine $\mathrm{Mo}_{2} \mathrm{Ti}_{2} \mathrm{C}_{3} \mathrm{~T}_{\mathrm{x}},(\mathrm{h})$ annealed $\mathrm{Mo}_{2} \mathrm{Ti}_{2} \mathrm{C}_{3} \mathrm{~T}_{\mathrm{x}}$.

structure of the ternary and quaternary carbides and separations between layers after chemical etching in the multilayer MXenes.

Figure 2(a) shows a digital photograph of $\mathrm{Mo}_{2} \mathrm{CT}_{\mathrm{x}}$, $\mathrm{Mo}_{2} \mathrm{TiC}_{2} \mathrm{~T}_{\mathrm{x}}$, and $\mathrm{Mo}_{2} \mathrm{Ti}_{2} \mathrm{C}_{3} \mathrm{~T}_{\mathrm{x}}$ freestanding papers, which were peeled off from a porous membrane after vacuum-assisted filtration and cut into $1 \times 1 \mathrm{~cm}^{2}$ squares for thermoelectric property measurements. Those freestanding paper samples are usually flexible in the pristine state without the use of binder additives, as shown in Figure 2(b). The flexibility can be altered upon annealing unless the nanosheets are supported by a flexible substrate. To achieve both high electrical conductivity and flexibility of MXene paper in pristine or mild dried state, cation intercalation can be used instead of organic intercalation as demonstrated in $\mathrm{Ti}_{3} \mathrm{C}_{2} \mathrm{~T}_{\mathrm{x}}$ MXene. ${ }^{4,}{ }^{11}$ Figure 2(c-h) show cross-sectional SEM micrographs of the as-synthesized Mo-based MXene papers (pristine) and after the thermoelectric measurements from room temperature (RT) to $800 \mathrm{~K}$ (annealed). The typical layered structure due to the restacking of MXene flakes is clearly observed in the pristine MXene papers. The cross-sectional SEM images of the annealed MXene papers show more compact structures, which lead to increased electrical conductivity, as will be discussed later.

X-ray diffraction (XRD) patterns of the parent layered carbides with corresponding MXene papers in pristine and annealed states are presented in Figure 3(a-c). Pristine MXene papers (middle patterns) show a large shift of the (002) peak toward lower angles compared to corresponding parent carbide precursor phases (bottom patterns). This result suggests an increase in the $c$-lattice parameter ( $c$-LP) in the MXene papers due to the surface functional groups replacing the 'Aelement' layers, as well as the presence of intercalated TBA ${ }^{+}$ and water molecules between the MXene nanosheets. ${ }^{37}$ After annealing the MXene papers at $800 \mathrm{~K}$ (top patterns), the (002) peak shifts back to higher angles, which can be explained by a decrease in the $c$-LP due to the removal of the intercalated molecules, $\mathrm{OH}$ and some other terminations. ${ }^{38}$ For $\mathrm{Mo}_{2} \mathrm{Ga}_{2} \mathrm{C}$ and its MXene (pristine and annealed, Figure 3(a)), the (002) peaks are centered at $9.76^{\circ}, 5.24^{\circ}$, and $11.17^{\circ}$, respectively. The corresponding $c$-LPs are $18.1 \AA, 33.7 \AA$, and $15.8 \AA$. The interlayer spacing between $\mathrm{Mo}_{2} \mathrm{C}$ layers in pristine $\mathrm{Mo}_{2} \mathrm{CT}_{\mathrm{x}}$ compared to $\mathrm{Mo}_{2} \mathrm{Ga}_{2} \mathrm{C}$ increased by $\sim 7.8 \AA$. In $\mathrm{Mo}_{2} \mathrm{Ga}_{2} \mathrm{C}$, two atomic layers of $\mathrm{Ga}$ separate the $\mathrm{Mo}_{2} \mathrm{C}$ layers. After etching and delamination, these Ga layers are replaced by surface terminations and intercalated $\mathrm{TBA}^{+}$and water molecules. After annealing the $\mathrm{Mo}_{2} \mathrm{CT}_{\mathrm{x}}$ paper, the interlayer spacing decreased by $\sim 8.9 \AA$, due to the removal of intercalated molecules and cations. $\mathrm{Mo}_{2} \mathrm{CT}_{\mathrm{x}}$ with no intercalated molecules has been calculated to have similar $c$-LP. ${ }^{39}$ Considering the synthesis process described above, one observes a large change in the interlayer distance, which is due to the intercalation (pristine MXene) and removal of organic molecules (annealed MXene). A similar trend can be found in $\mathrm{Mo}_{2} \mathrm{TiAlC}_{2}$ (Figure 3(b)), where the $c$-LP value first increases from $18.5 \AA$ for the precursor $\left(\mathrm{Mo}_{2} \mathrm{TiAlC}_{2}\right)$ to $37.8 \AA$ after etching the $\mathrm{Al}$ layers and intercalation, and decreases to $24.5 \AA$ due to the removal of intercalated organic and water molecules during heating to $800 \mathrm{~K}$. In the case of $\mathrm{Mo}_{2} \mathrm{Ti}_{2} \mathrm{AlC}_{3}$ (Figure 3(c)), the initial cLP value is $23.4 \AA$ based on the (004) peak position at $15.13^{\circ}$ for the $\mathrm{Mo}_{2} \mathrm{Ti}_{2} \mathrm{AlC}_{3}$, and it increases to $42.6 \AA$ for the pristine $\mathrm{Mo}_{2} \mathrm{Ti}_{2} \mathrm{C}_{3} \mathrm{~T}_{\mathrm{x}}$ paper and decreases to $27.6 \AA$ after annealing. The crystallographic ionic diameter of $\mathrm{TBA}^{+}$is known to be around $9.9 \AA$, however a minor distortion/conformation of butyl chains allows the intercalation into $8 \AA$ interlayer space. ${ }^{40}$ Thus, the observed shifts for the basal plane related peak positions correlate with the intercalation and deintercalation of $\mathrm{TBA}^{+}$and water molecules.

Figure 3(d-f) shows the Raman spectra of three Mo-based layered carbide precursors (bottom) and their corresponding pristine (middle) and annealed MXenes (top). There are clear changes in the Raman spectra after etching that show disappearance and/or shift of the Raman active vibration modes 

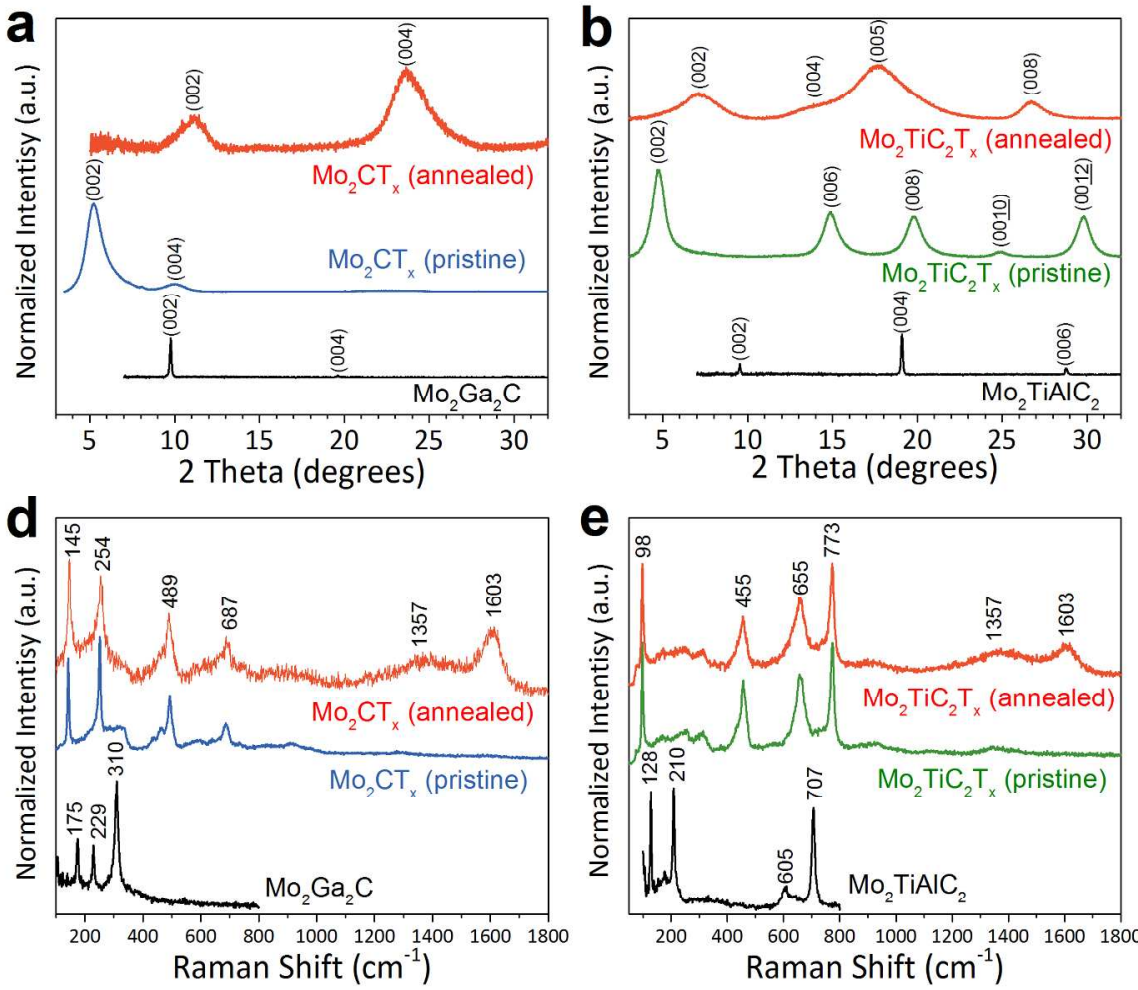
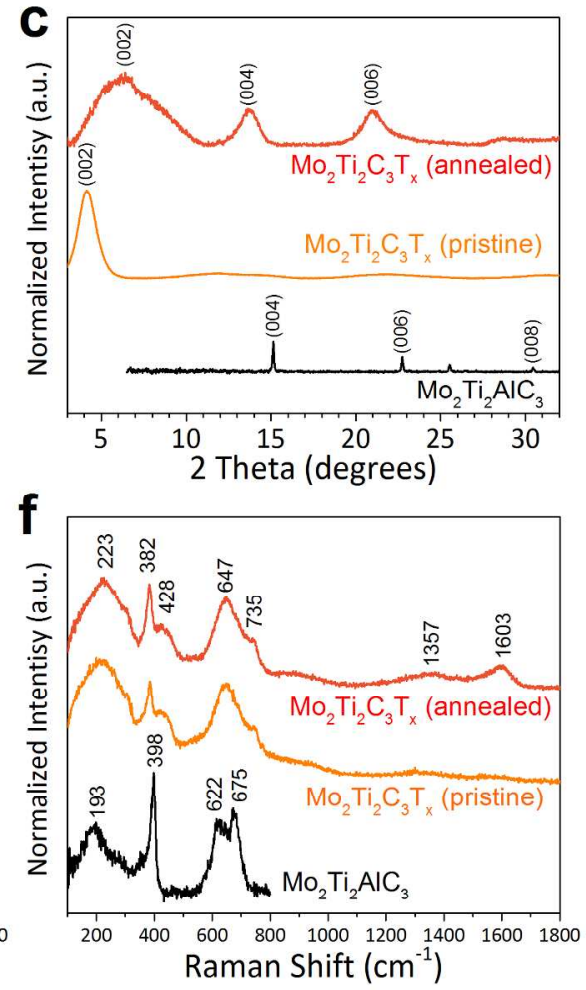

Figure 3. (a-c) X-ray diffraction patterns and (d-f) Raman spectra of the parent layered carbide phases and corresponding pristine and annealed MXene papers. (a,d) $\mathrm{Mo}_{2} \mathrm{Ga}_{2} \mathrm{C}$ and $\mathrm{Mo}_{2} \mathrm{CT}_{\mathrm{x}}$ (b,e) $\mathrm{Mo}_{2} \mathrm{TiAlC}_{2}$ and $\mathrm{Mo}_{2} \mathrm{TiC}_{2} \mathrm{~T}_{\mathrm{x}}$, (c,f) $\mathrm{Mo}_{2} \mathrm{Ti}_{2} \mathrm{AlC}_{3}$ and $\mathrm{Mo}_{2} \mathrm{Ti}_{2} \mathrm{C}_{3} \mathrm{~T}_{\mathrm{x}}$. There are no XRD peaks below 8 degree in patterns of precursors. There are no Raman peaks above $800 \mathrm{~cm}^{-1}$ in the spectra of precursors.

related to M-A vibrations, and appearance of new modes which may be related to surface functionalization and/or additional modes becoming active in $2 \mathrm{D}$ layers, when the constraint of M-A bonding in the 3D structure has been removed. Weak carbon bands appeared after annealing, suggesting either formation of disordered carbon due to unavoidable carbon contamination such as carbon dioxide, or some disproportionation of MXene possibly due to partial loss of surface terminations. ${ }^{41}$ All peaks of MXene stay in place and peak shifts are minor, indicating that the structure of MXene was retained after annealing and the reported properties are representative of the MXenes, not their decomposition products. Our experimental Raman spectra are in good agreement with multiple simulation studies using O termination. See Supporting Information for the detailed interpretation of Raman spectra of MXenes and their precursors.

Temperature dependent thermoelectric properties of the Mobased MXenes are presented in Figure 4(a-c). All three MXene papers are highly resistive in the pristine state near RT, but their electrical conductivity increases rapidly during the first heating cycle, with three identifiable regimes: (1) Removal of water molecules gradually enhances the conductivity at low temperatures $(300-500 \mathrm{~K})$. (2) Thermal decomposition of $\mathrm{TBA}^{+}$intercalant at around $500-600 \mathrm{~K}$. The onset of rapid conductivity increase is well matched to the reported thermal decomposition temperature of $\mathrm{TBA}^{+}$intercalant in graphite, which is around $480 \mathrm{~K}^{40}(3)$ Above $600 \mathrm{~K}$, the conductivity increases slowly which can be ascribed to the decomposition of remaining $\mathrm{TBA}^{+}$and the possible thermal elimination of surface termination groups. It should be noted that the observed temperature dependent electrical conductivity during the $1^{\text {st }}$ heating cycle is the sum of both irreversible changes due to thermal de-intercalation, and reversible thermal response of the material itself, where the former factor is more dominant. The MXene flakes are likely to undergo rearrangement throughout the thermal heating process described above, where the interlayer spacing decreases. In order to confirm the above hypothesis about the cause of the conductivity change during the first heating cycle in MXenes, thermal gravimetric analysis (TGA) was carried out along with differential scanning calorimetry (DSC) for $\mathrm{Mo}_{2} \mathrm{TiC}_{2} \mathrm{~T}_{\mathrm{x}}$ paper, as shown in Figure 4(d). An overall weight loss of around $8 \%$ was observed from RT to $800 \mathrm{~K}$ under Ar ambient. The quantity of intercalated water molecules in pristine $\mathrm{Mo}_{2} \mathrm{TiC}_{2} \mathrm{~T}_{\mathrm{x}}$ is estimated around $1.6 \mathrm{wt} \%$ based on the weight loss below $500 \mathrm{~K}^{42}$ Most of weight loss (5.3 wt\%) between $500-650 \mathrm{~K}$ can be attributed to the thermal de-intercalation of $\mathrm{TBA}^{+}$, and this temperature range matches the one in which electrical conductivity of MXenes shows a significant increase. This result suggests that the MXene flakes in the pristine paper are separated by large intercalant molecules, but come in closer contact that enhances the carrier transport due to the thermal deintercalation process at around $500-600 \mathrm{~K}$. These changes, also supported by XRD studies (Figure 3(a-c)), are irreversible and are not observed in the $2^{\text {nd }}$ thermal cycle of TGADSC, nor during the $2^{\text {nd }}$ thermal cycle of thermoelectric properties measurement (Figures S3-S5). At above $650 \mathrm{~K}$, only $\sim 1$ wt $\%$ of weight loss was observed, while the electrical conductivity slowly increased. Despite the small changes in both weight and electrical conductivity above $650 \mathrm{~K}$, relatively 


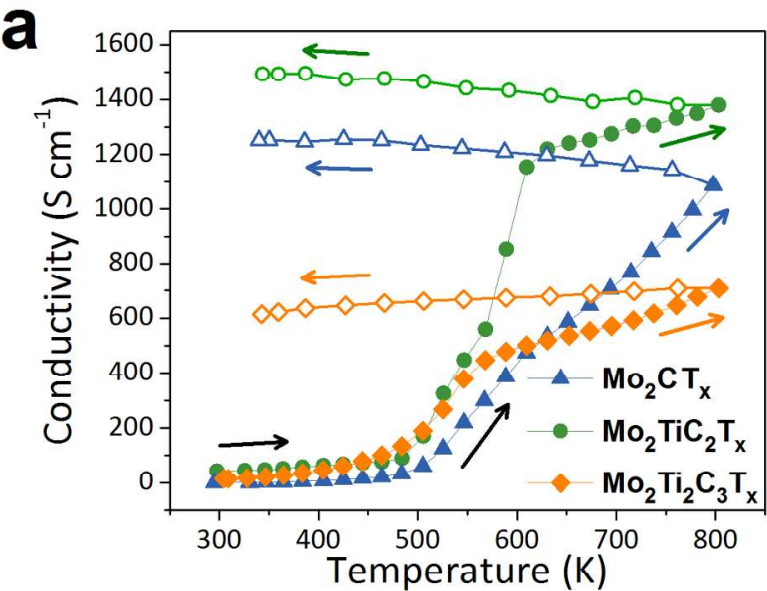

b

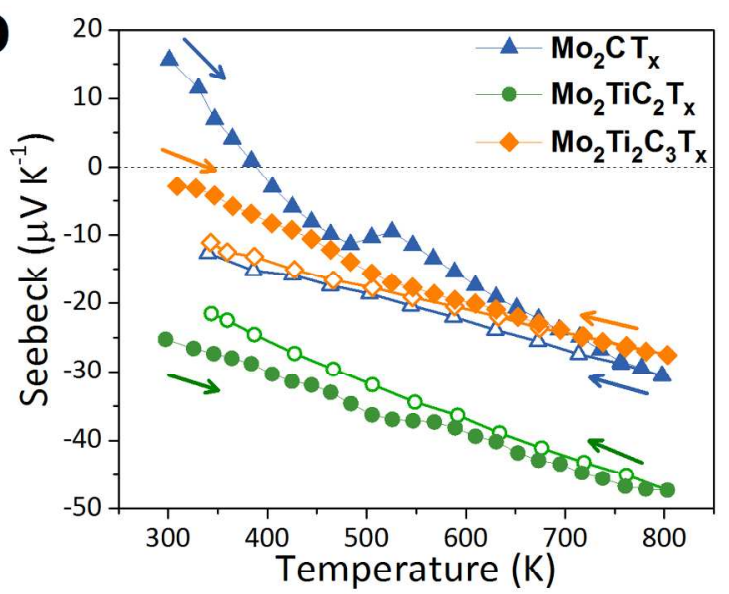

C

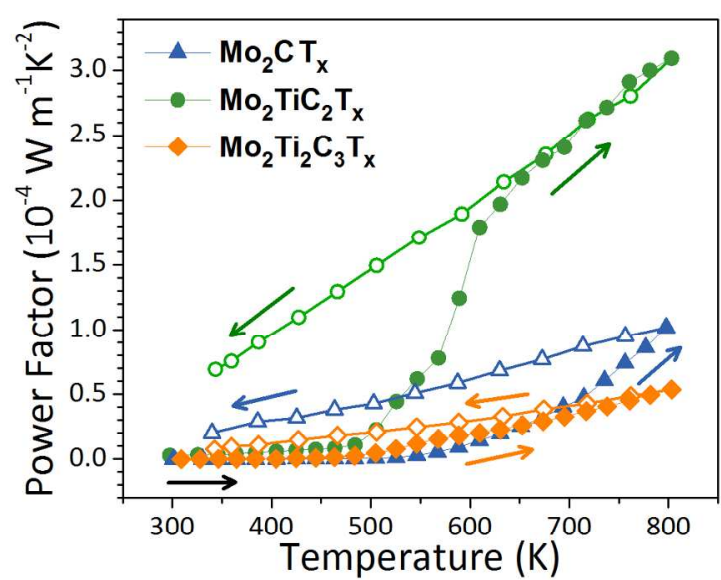

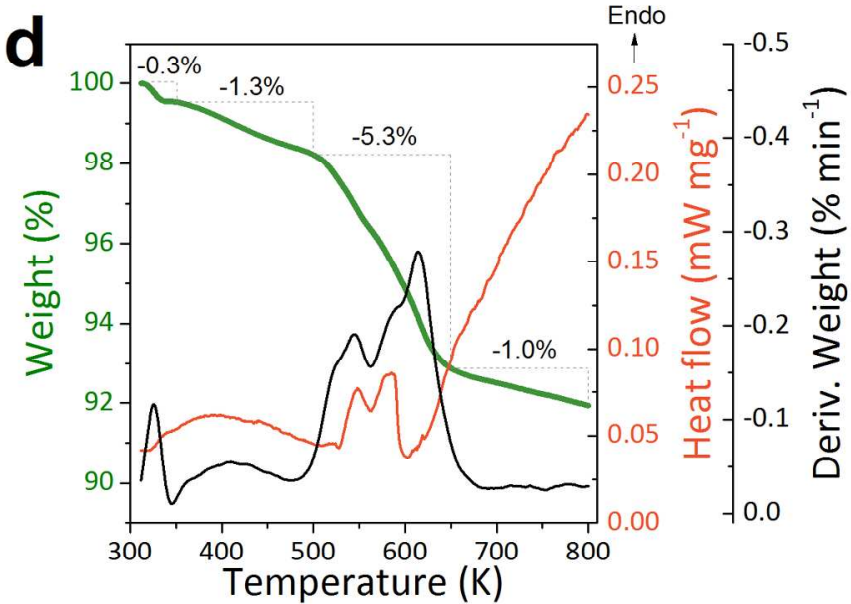

Figure 4. (a-c) Temperature dependent thermoelectric properties of Mo-based MXene papers during the first thermal cycle. (a) Electrical conductivity, (b) Seebeck coefficient, and (c) thermoelectric power factor. The heating cycle and subsequent cooling cycle are marked by filled symbols and open symbols, respectively. The blue triangle, green circle, orange diamond symbols represent $\mathrm{Mo}_{2} \mathrm{CT}_{\mathrm{x}}, \mathrm{Mo}_{2} \mathrm{TiC}_{2} \mathrm{~T}_{\mathrm{x}}$, and $\mathrm{Mo}_{2} \mathrm{Ti}_{2} \mathrm{C}_{3} \mathrm{~T}_{\mathrm{x}}$, respectively. (d) TGA curve (green) with first derivative of weight (black) and $\mathrm{DSC}$ curve (red) of $\mathrm{Mo}_{2} \mathrm{TiC}_{2} \mathrm{~T}_{\mathrm{x}}$ for the first heating cycle.

large endothermic heat flow was observed during both the first and second thermal cycle. Considering that the weight change in second thermal cycle is nearly zero (Figure S4(d)), the heat could be absorbed due to the removal of surface functional groups, as earlier observation for $\mathrm{Ti}_{3} \mathrm{C}_{2} \mathrm{~T}_{\mathrm{x}}{ }^{38}$ The $c$-LP of $\mathrm{Ti}_{3} \mathrm{C}_{2} \mathrm{~T}_{\mathrm{x}}$ without intercalated water molecule decreased by only $0.5 \AA$ after annealing up to $773 \mathrm{~K}$ under vacuum. ${ }^{43}$ Since the surface functional groups are known to accept electrons from MXenes, ${ }^{44}$ removal of surface terminations can be another contributor to the increase in electrical conductivity.

The electrical conductivity of pristine $\mathrm{Mo}_{2} \mathrm{CT}_{\mathrm{x}}$ measured before annealing is $0.8 \mathrm{~S} \mathrm{~cm}^{-1}$, but increases to $1252 \mathrm{~S} \mathrm{~cm}^{-1}$ at the end of the first thermal cycle (heating and then cooling to near RT) during thermoelectric measurements. This result is in agreement with literature, where the RT electrical conductivity of $\mathrm{Mo}_{2} \mathrm{CT}_{\mathrm{x}}$ was shown to increase by two orders of magnitude after drying at $120^{\circ} \mathrm{C}$ for 18 hours under vacuum. ${ }^{32}$ The conductivity values of pristine (annealed) $\mathrm{Mo}_{2} \mathrm{TiC}_{2} \mathrm{~T}_{\mathrm{x}}$ and $\mathrm{Mo}_{2} \mathrm{Ti}_{2} \mathrm{C}_{3} \mathrm{~T}_{\mathrm{x}}$, are $44 \mathrm{~S} \mathrm{~cm}^{-1}\left(1494 \mathrm{~S} \mathrm{~cm}^{-1}\right)$ and $15 \mathrm{~S} \mathrm{~cm}^{-1}(614 \mathrm{~S}$ $\left.\mathrm{cm}^{-1}\right)$, respectively. Table 1 shows a summary of the Halleffect measurement results. Pristine Mo-based MXene papers typically have carrier density of $\sim 10^{20} \mathrm{~cm}^{-3}$ with a Hall mobility of $10^{-2} \sim 10^{-1} \mathrm{~cm}^{2} \mathrm{~V}^{-1} \mathrm{~s}^{-1}$. Annealed Mo-based MXene papers exhibit one order of magnitude higher carrier concentration with Hall mobility of few $\mathrm{cm}^{2} \mathrm{~V}^{-1} \mathrm{~s}^{-1}$. The changes in conductivity during the first annealing are attributed to the decreased interlayer spacing due to thermal de-intercalation and partial de-functionalization of MXenes. Once the conductivity stabilizes after the first heating cycle, $\mathrm{Mo}_{2} \mathrm{CT}_{\mathrm{x}}$ and $\mathrm{Mo}_{2} \mathrm{TiC}_{2} \mathrm{~T}_{\mathrm{x}}$ exhibit metallic behavior while $\mathrm{Mo}_{2} \mathrm{Ti}_{2} \mathrm{C}_{3} \mathrm{~T}_{\mathrm{x}}$ shows semiconductor-like behavior, based on the slope of temperature dependence of conductivity in the stabilized (annealed) state. These characteristics are related to the carrier concentration and may have also been affected by the stacking structure, where annealed $\mathrm{Mo}_{2} \mathrm{CT}_{\mathrm{x}}$ and $\mathrm{Mo}_{2} \mathrm{TiC}_{2} \mathrm{~T}_{\mathrm{x}}$ show well aligned nanosheets (Figure 2(d,f)), while annealed $\mathrm{Mo}_{2} \mathrm{Ti}_{2} \mathrm{C}_{3} \mathrm{~T}_{\mathrm{x}}$ films look less compacted (Figure 2(h)). Larger thickness of $\mathrm{Mo}_{2} \mathrm{Ti}_{2} \mathrm{C}_{3} \mathrm{~T}_{\mathrm{x}}$ nanosheets could make their rearrangement and film densification more difficult, but we did not find a good way to quantify those differences. 
TABLE 1. Hall-effect measurement results of Mo-based MXene papers at RT. ${ }^{\text {a) }}$

\begin{tabular}{|l|l|l|l|}
\hline Sample & $\boldsymbol{n}\left(\mathbf{1} / \mathbf{c m}^{\mathbf{3}}\right)$ & $\boldsymbol{\mu}\left(\mathbf{c m}^{\mathbf{2}} / \mathbf{V} \cdot \mathbf{s}\right)$ & Type \\
\hline $\mathrm{Mo}_{2} \mathrm{CT}_{\mathrm{x}}$ (pristine) & $2.84 \times 10^{20}$ & 0.016 & $\mathrm{p}$ \\
\hline $\mathrm{Mo}_{2} \mathrm{TiC}_{2} \mathrm{~T}_{\mathrm{x}}$ (pristine) & $8.31 \times 10^{20}$ & 0.323 & $\mathrm{n}$ \\
\hline $\mathrm{Mo}_{2} \mathrm{Ti}_{2} \mathrm{C}_{3} \mathrm{~T}_{\mathrm{x}}$ (pristine) & $2.01 \times 10^{20}$ & 0.451 & $\mathrm{n}$ \\
\hline $\mathrm{Mo}_{2} \mathrm{CT}_{\mathrm{x}}$ (annealed) & $4.10 \times 10^{21}$ & 1.86 & $\mathrm{n}$ \\
\hline $\mathrm{Mo}_{2} \mathrm{TiC}_{2} \mathrm{~T}_{\mathrm{x}}$ (annealed) & $3.04 \times 10^{21}$ & 2.85 & $\mathrm{n}$ \\
\hline $\mathrm{Mo}_{2} \mathrm{Ti}_{2} \mathrm{C}_{3} \mathrm{~T}_{\mathrm{x}}$ (annealed) & $1.83 \times 10^{21}$ & 2.05 & $\mathrm{n}$ \\
\hline
\end{tabular}

a) Carrier concentration $(n)$ and Hall mobility $(\mu)$.

The Seebeck coefficient of these MXenes were simultaneously measured as shown in Figure 4(b). All three MXenes show ntype behavior, as indicated by the negative values of the Seebeck coefficients. Unlike the rapid change in electrical conductivity, the Seebeck coefficient does not show a drastic transition; instead it undergoes a slow change during the first heating cycle. This observation is another evidence that supports the fact that the rapid increase in the electrical conductivity above $\sim 500 \mathrm{~K}$ (Figure 4(a)) is likely due to the enhanced electrical network between MXene nanosheets rather than irreversible changes of each MXene flakes. As the nanosheets get better connection during the first heating cycle, the energy barrier between MXene flakes will spontaneously decrease, hence a larger numbers of domains will contribute to its overall Seebeck coefficient. Particularly, $\mathrm{Mo}_{2} \mathrm{CT}_{\mathrm{x}}$ samples shows an interesting transition from p-type to n-type upon thermal annealing, as confirmed by both Hall-effect measurement and thermoelelctric test. The spontaneous transition of its Seebeck coefficient seems to be a result of a decreased energy barrier at the edges of MXene flakes during the first heating cycle.

Just like electrical conductivity, the Seebeck coefficient of all three MXenes reaches a steady-state value and remains stable after the first heating cycle, as shown in Figures S3-S5. The temperature dependent Seebeck coefficient is quite linear with temperature, indicating absence of any magnetic transition. $\mathrm{Mo}_{2} \mathrm{Ti}_{2} \mathrm{C}_{3} \mathrm{~T}_{\mathrm{x}}$ exhibits the smallest Seebeck coefficient, among the three samples, of $-27.5 \mu \mathrm{V} \mathrm{K}^{-1}$ at $803 \mathrm{~K}$, while $\mathrm{Mo}_{2} \mathrm{CT}_{\mathrm{x}}$ exhibits $-30.5 \mu \mathrm{V} \mathrm{K}^{-1}$ at $798 \mathrm{~K}$. These two compositions have similar temperature dependent Seebeck coefficient. $\mathrm{Mo}_{2} \mathrm{CF}_{2}$ has been theoretically predicted to be semiconducting with a large Seebeck coefficient $\left(>100 \mu \mathrm{V} \mathrm{K}^{-1}\right.$ at $\left.400 \mathrm{~K}\right)$ and a negative correlation between Seebeck coefficient and temperature. ${ }^{22}$ However, our experimental results show the opposite. This suggests that the prepared $\mathrm{Mo}_{2} \mathrm{CT}_{\mathrm{x}}$ may not be dominated by fluorine termination, despite the fact that we used an HF etching method. It has been reported that delamination of Mo-containing MXenes with TBAOH reduces the fluorine content. ${ }^{26}$ In contrast, $\mathrm{Mo}_{2} \mathrm{TiC}_{2} \mathrm{~T}_{\mathrm{x}}$ exhibited a larger Seebeck coefficient of $-47.3 \mu \mathrm{V} \mathrm{K}^{-1}$ at $803 \mathrm{~K}$, along with the largest slope. It is worth to note that the observed Seebeck coefficient of $\mathrm{Mo}_{2} \mathrm{TiC}_{2} \mathrm{~T}_{\mathrm{x}}$ MXene paper is higher than the theoretically calculated value of its parent $\mathrm{Mo}_{2} \mathrm{TiAlC}_{2} \mathrm{MAX}$ phase. $^{45}$

Due to its high conductivity $\left(1380 \mathrm{~S} \mathrm{~cm}^{-1}\right.$ at $\left.803 \mathrm{~K}\right)$ and large Seebeck coefficient $\left(-47.3 \mu \mathrm{V} \mathrm{K}^{-1}\right.$ at $\left.803 \mathrm{~K}\right), \mathrm{Mo}_{2} \mathrm{TiC}_{2} \mathrm{~T}_{\mathrm{x}}$ shows the largest thermoelectric power factor of $3.09 \times 10^{-4} \mathrm{~W} \mathrm{~m}^{-1} \mathrm{~K}^{-2}$ at $803 \mathrm{~K}$ among the MXenes studied here, as shown in Figure 4(c). $\mathrm{Mo}_{2} \mathrm{TiC}_{2} \mathrm{~T}_{\mathrm{x}}$ also shows nearly one order of magnitude higher power factor near RT compared to chemically exfoliated $\mathrm{WS}_{2}$, which has been demonstrated as a wearable thermoelectric generator. ${ }^{46}$ This superior thermoelectric power factor of $\mathrm{Mo}_{2} \mathrm{TiC}_{2} \mathrm{~T}_{\mathrm{x}}$ can be attributed to its electronic band structure. One possible explanation is the so-called "pudding-mold" type band structure found in high performance thermoelectric materials such as $\mathrm{Na}_{\mathrm{x}} \mathrm{CoO}_{2}$ and $\mathrm{CuAlO}_{2}{ }^{47,}{ }^{48}$ The band near the Fermi level can be flatter depending on the ratio of hopping integrals to the first, second, and third nearest neighbor sites on the triangular lattice, ${ }^{48,}{ }^{49}$ where the peculiar shape band structure can result multiple Fermi surfaces that can contribute to the conductivity overcoming the decrease in Seebeck coefficient. Such variable range hopping behavior can be related to the geometrical frustration in triangular lattice with the antiferromagnetic spin interaction. However, more theoretical work is needed to confirm this hypothesis. According to the simulation studies on the electronic band structure of Mo-based MXenes with oxygen termination, ${ }^{27,}{ }^{28,50} \mathrm{Mo}_{2} \mathrm{TiC}_{2} \mathrm{O}_{2}$ certainly shows a much larger portion of flat band near the Fermi level than $\mathrm{Mo}_{2} \mathrm{CO}_{2}$ and $\mathrm{Mo}_{2} \mathrm{Ti}_{2} \mathrm{C}_{3} \mathrm{O}_{2}$, which can be the origin of coexistence of metallic conductivity and a relatively larger Seebeck coefficient. Due to the highly conductive nature of MXenes, hybrid materials with outstanding thermoelectric performance may be produced by combining MXenes with large band gap semiconductors to increase the overall Seebeck coefficient and/or polymers to decrease the thermal conductivity. $^{51,52}$

\section{Conclusion}

In summary, we have reported the first experimental measurements of the temperature dependent thermoelectric properties of Mo-based MXenes up to $800 \mathrm{~K}$. A combination of a high electrical conductivity $\left(1380 \mathrm{~S} \mathrm{~cm}^{-1}\right.$ at $\left.803 \mathrm{~K}\right)$ and a relatively large Seebeck coefficient $\left(-47.3 \mu \mathrm{V} \mathrm{K}^{-1}\right.$ at $\left.803 \mathrm{~K}\right)$ has been found for $\mathrm{Mo}_{2} \mathrm{TiC}_{2} \mathrm{~T}_{\mathrm{x}}$, with its thermoelectric power factor reaching $3.09 \times 10^{-4} \mathrm{~W} \mathrm{~m}^{-1} \mathrm{~K}^{-2}$ at $803 \mathrm{~K}$. The conductivity of all MXenes studied in this work shows a rapid increase above $\sim 500 \mathrm{~K}$, which is due to de-intercalation of water and organic molecules, as well as partial loss of functional groups resulting in a decrease of interlayer spacing and improved contacts between the MXene nanosheets. Raman spectroscopy analysis revealed that Mo-based MXenes are thermally robust upon annealing at up to $800 \mathrm{~K}$ under $\mathrm{Ar} / \mathrm{H}_{2}$ ambient. Further enhancement in thermoelectric performance of MXenes can be achieved by additional doping, controlling the surface functional groups, and/or hybridization with semiconductor materials or polymers.

\section{ASSOCIATED CONTENT}

Supporting Information. SEM images of precursor materials and HF-etched multilayer MXenes; Extended Raman spectra of annealed MXene papers; Thermoelectric data for $2^{\text {nd }}$ thermal cycle; Raman spectroscopy analysis of Mo-based MXenes. This material is available free of charge via the Internet at http://pubs.acs.org.

\section{AUTHOR INFORMATION}

\section{Corresponding Author}

*E-mail: husam.alshareef@kaust.edu.sa. 


\section{Author Contributions}

All authors have given approval to the final version of the manuscript.

\section{Funding Sources}

Support for research reported in this publication was provided by King Abdullah University of Science and Technology (KAUST).

\section{Notes}

The authors declare no competing financial interest.

\section{ACKNOWLEDGMENT}

The authors thank Dr. Narendra Kurra for useful discussions and Dr. Rajeshkumar Mohanraman for technical help in TGA-DSC analysis.

\section{REFERENCES}

(1) Naguib, M.; Kurtoglu, M.; Presser, V.; Lu, J.; Niu, J. J.; Heon, M.; Hultman, L.; Gogotsi, Y.; Barsoum, M. W., Two-Dimensional Nanocrystals Produced by Exfoliation of $\mathrm{Ti}_{3} \mathrm{AlC}_{2}$. Adv. Mater. 2011, 23, 4248-4253.

(2) Anasori, B.; Lukatskaya, M. R.; Gogotsi, Y., 2D Metal Carbides and Nitrides (MXenes) for Energy Storage. Nat. Rev. Mater. 2017, 2, 16098 .

(3) Tao, Q. Z.; Dahlqvist, M.; Lu, J.; Kota, S.; Meshkian, R.; Halim, J.; Palisaitis, J.; Hultman, L.; Barsoum, M. W.; Persson, P. O. A.; Rosen, J., Two-Dimensional $\mathrm{Mo}_{1.33} \mathrm{C}$ MXene with Divacancy Ordering Prepared from Parent 3D Laminate with In-plane Chemical Ordering. Nat. Commun. 2017, 8, 14949.

(4) Lukatskaya, M. R.; Mashtalir, O.; Ren, C. E.; Dall'Agnese, Y.; Rozier, P.; Taberna, P. L.; Naguib, M.; Simon, P.; Barsoum, M. W.; Gogotsi, Y., Cation Intercalation and High Volumetric Capacitance of Two-Dimensional Titanium Carbide. Science 2013, 341, 1502-1505.

(5) Naguib, M.; Halim, J.; Lu, J.; Cook, K. M.; Hultman, L.; Gogotsi, Y.; Barsoum, M. W., New Two-Dimensional Niobium and Vanadium Carbides as Promising Materials for Li-Ion Batteries. $J$. Am. Chem. Soc. 2013, 135, 15966-15969.

(6) Ghidiu, M.; Lukatskaya, M. R.; Zhao, M. Q.; Gogotsi, Y.; Barsoum, M. W., Conductive Two-Dimensional Titanium Carbide 'Clay' with High Volumetric Capacitance. Nature 2014, 516, 78-81.

(7) Ling, Z.; Ren, C. E.; Zhao, M. Q.; Yang, J.; Giammarco, J. M.; Qiu, J. S.; Barsoum, M. W.; Gogotsi, Y., Flexible and Conductive MXene Films and Nanocomposites with High Capacitance. Proc. Natl. Acad. Sci. U.S.A. 2014, 111, 16676-16681.

(8) Mashtalir, O.; Lukatskaya, M. R.; Zhao, M. Q.; Barsoum, M. W.; Gogotsi, Y., Amine-Assisted Delamination of $\mathrm{Nb}_{2} \mathrm{C}$ MXene for Li-Ion Energy Storage Devices. Adv. Mater. 2015, 27, 3501-3506.

(9) Zhao, M. Q.; Ren, C. E.; Ling, Z.; Lukatskaya, M. R.; Zhang, C. F.; Van Aken, K. L.; Barsoum, M. W.; Gogotsi, Y., Flexible MXene/Carbon Nanotube Composite Paper with High Volumetric Capacitance. Adv. Mater. 2015, 27, 339-345.

(10) Tang, Q.; Zhou, Z.; Shen, P. W., Are MXenes Promising Anode Materials for Li Ion Batteries? Computational Studies on Electronic Properties and Li Storage Capability of $\mathrm{Ti}_{3} \mathrm{C}_{2}$ and $\mathrm{Ti}_{3} \mathrm{C}_{2} \mathrm{X}_{2}(\mathrm{X}=$ F, OH) Monolayer. J. Am. Chem. Soc. 2012, 134, 16909-16916.

(11) Shahzad, F.; Alhabeb, M.; Hatter, C. B.; Anasori, B.; Hong, S. M.; Koo, C. M.; Gogotsi, Y., Electromagnetic Interference Shielding with 2D Transition Metal Carbides (MXenes). Science 2016, 353, 1137-1140.

(12) Xu, B. Z.; Zhu, M. S.; Zhang, W. C.; Zhen, X.; Pei, Z. X.; Xue, Q.; Zhi, C. Y.; Shi, P., Ultrathin MXene-Micropattern-Based Field-Effect Transistor for Probing Neural Activity. Adv. Mater. 2016, 28, 3333-3339.

(13) Liu, H.; Duan, C. Y.; Yang, C. H.; Shen, W. Q.; Wang, F.; Zhu, Z. F., A Novel Nitrite Biosensor Based on the Direct Electrochemistry of Hemoglobin Immobilized on MXene-Ti ${ }_{3} \mathrm{C}_{2}$. Sens. Actuators, $B$ 2015, 218, 60-66.
(14) Wang, F.; Yang, C. H.; Duan, C. Y.; Xiao, D.; Tang, Y.; Zhu, J. F., An Organ-Like Titanium Carbide Material (MXene) with Multilayer Structure Encapsulating Hemoglobin for a Mediator-Free Biosensor. J. Electrochem. Soc. 2015, 162, B16-B21.

(15) Ren, C. E.; Hatzell, K. B.; Alhabeb, M.; Ling, Z.; Mahmoud, K. A.; Gogotsi, Y., Charge- and Size-Selective Ion Sieving Through $\mathrm{Ti}_{3} \mathrm{C}_{2} \mathrm{~T}_{\mathrm{x}}$ MXene Membranes. J. Phys. Chem. Lett. 2015, 6, 40264031.

(16) Zhang, Q. R.; Teng, J.; Zou, G. D.; Peng, Q. M.; Du, Q.; Jiao, T. F.; Xiang, J. Y., Efficient Phosphate Sequestration for Water Purification by Unique Sandwich-Like MXene/Magnetic Iron Oxide Nanocomposites. Nanoscale 2016, 8, 7085-7093.

(17) Rasool, K.; Helal, M.; Ali, A.; Ren, C. E.; Gogotsi, Y.; Mahmoud, K. A., Antibacterial Activity of $\mathrm{Ti}_{3} \mathrm{C}_{2} \mathrm{~T}_{\mathrm{x}}$ MXene. ACS Nano 2016, 10, 3674-3684.

(18) Lei, J. C.; Zhang, X.; Zhou, Z., Recent Advances in MXene: Preparation, Properties, and Applications. Front. Phys. 2015, 10, 276286.

(19) Khazaei, M.; Arai, M.; Sasaki, T.; Chung, C. Y.; Venkataramanan, N. S.; Estili, M.; Sakka, Y.; Kawazoe, Y., Novel Electronic and Magnetic Properties of Two-Dimensional Transition Metal Carbides and Nitrides. Adv. Funct. Mater. 2013, 23, 2185-2192.

(20) Gandi, A. N.; Alshareef, H. N.; Schwingenschlogl, U., Thermoelectric Performance of the MXenes $\mathrm{M}_{2} \mathrm{CO}_{2}(\mathrm{M}=\mathrm{Ti}, \mathrm{Zr}$, or Hf). Chem. Mater. 2016, 28, 1647-1652.

(21) Kumar, S.; Schwingenschlogl, U., Thermoelectric Performance of Functionalized $\mathrm{Sc}_{2} \mathrm{C}$ MXenes. Phys. Rev. B 2016, 94, 035405 .

(22) Khazaei, M.; Arai, M.; Sasaki, T.; Estili, M.; Sakka, Y., TwoDimensional Molybdenum Carbides: Potential Thermoelectric Materials of the MXene Family. Phys. Chem. Chem. Phys. 2014, 16, 78417849.

(23) Hope, M. A.; Forse, A. C.; Griffith, K. J.; Lukatskaya, M. R.; Ghidiu, M.; Gogotsi, Y.; Grey, C. P., NMR Reveals the Surface Functionalisation of $\mathrm{Ti}_{3} \mathrm{C}_{2}$ MXene. Phys. Chem. Chem. Phys. 2016, 18, 5099-5102.

(24) Anasori, B.; Xie, Y.; Beidaghi, M.; Lu, J.; Hosler, B. C.; Hultman, L.; Kent, P. R. C.; Gogotsi, Y.; Barsoum, M. W., TwoDimensional, Ordered, Double Transition Metals Carbides (MXenes). ACS Nano 2015, 9, 9507-9516.

(25) Bai, Y. L.; He, X. D.; Wang, R. G., Lattice Dynamics of AlContaining MAX-Phase Carbides: A First-Principle Study. J. Raman Spectrosc. 2015, 46, 784-794.

(26) Anasori, B.; Shi, C.; Moon, E. J.; Xie, Y.; Voigt, C. A.; Kent, P. R. C.; May, S. J.; Billinge, S. J. L.; Barsoum, M. W.; Gogotsi, Y., Control of Electronic Properties of 2D Carbides (MXenes) by Manipulating Their Transition Metal Layers. Nanoscale Horiz. 2016, 1, 227-234.

(27) Weng, H. M.; Ranjbar, A.; Liang, Y. Y.; Song, Z. D.; Khazaei, M.; Yunoki, S.; Arai, M.; Kawazoe, Y.; Fang, Z.; Dai, X., Large-Gap Two-Dimensional Topological Insulator in Oxygen Functionalized MXene. Phys. Rev. B 2015, 92, 075436.

(28) Khazaei, M.; Ranjbar, A.; Arai, M.; Yunoki, S., Topological Insulators in the Ordered Double Transition Metals $\mathrm{M}_{2}{ }^{\prime} \mathrm{M}^{\prime \prime} \mathrm{C}_{2}$ MXenes ( $\left.\mathrm{M}^{\prime}=\mathrm{Mo}, \mathrm{W} ; \mathrm{M}^{\prime \prime}=\mathrm{Ti}, \mathrm{Zr}, \mathrm{Hf}\right)$. Phys. Rev. B 2016, 94, 125152.

(29) Si, C.; Jin, K. H.; Zhou, J.; Sun, Z. M.; Liu, F., Large-Gap Quantum Spin Hall State in MXenes: d-Band Topological Order in a Triangular Lattice. Nano Lett. 2016, 16, 6584-6591.

(30) Xu, Y.; Gan, Z. X.; Zhang, S. C., Enhanced Thermoelectric Performance and Anomalous Seebeck Effects in Topological Insulators. Phys. Rev. Lett. 2014, 112, 226801.

(31) Chen, Y. L.; Analytis, J. G.; Chu, J. H.; Liu, Z. K.; Mo, S. K.; Qi, X. L.; Zhang, H. J.; Lu, D. H.; Dai, X.; Fang, Z.; Zhang, S. C.; Fisher, I. R.; Hussain, Z.; Shen, Z. X., Experimental Realization of a Three-Dimensional Topological Insulator, $\mathrm{Bi}_{2} \mathrm{Te}_{3}$. Science 2009, 325 , 178-181.

(32) Halim, J.; Kota, S.; Lukatskaya, M. R.; Naguib, M.; Zhao, M. Q.; Moon, E. J.; Pitock, J.; Nanda, J.; May, S. J.; Gogotsi, Y.; Bar- 
soum, M. W., Synthesis and Characterization of 2D Molybdenum Carbide (MXene). Adv. Funct. Mater. 2016, 26, 3118-3127.

(33) Kim, H.; Wang, Z. W.; Hedhili, M. N.; Wehbe, N.; Alshareef, H. N., Oxidant-Dependent Thermoelectric Properties of Undoped ZnO Films by Atomic Layer Deposition. Chem. Mater. 2017, 29, 2794-2802.

(34) Hu, C.; Lai, C. C.; Tao, Q.; Lu, J.; Halim, J.; Sun, L.; Zhang, J.; Yang, J.; Anasori, B.; Wang, J.; Sakka, Y.; Hultman, L.; Eklund, P.; Rosen, J.; Barsoum, M. W., $\mathrm{Mo}_{2} \mathrm{Ga}_{2} \mathrm{C}$ : A New Ternary Nanolaminated Carbide. Chem. Commun. 2015, 51, 6560-6563.

(35) Anasori, B.; Halim, J.; Lu, J.; Voigt, C. A.; Hultman, L.; Barsoum, M. W., $\mathrm{Mo}_{2} \mathrm{TiAlC}_{2}$ : A New Ordered Layered Ternary Carbide. Scripta Mater. 2015, 101, 5-7.

(36) Anasori, B.; Dahlqvist, M.; Halim, J.; Moon, E. J.; Lu, J.; Hosler, B. C.; Caspi, E. N.; May, S. J.; Hultman, L.; Eklund, P.; Rosen, J.; Barsoum, M. W., Experimental and Theoretical Characterization of Ordered MAX Phases $\mathrm{Mo}_{2} \mathrm{TiAlC}_{2}$ and $\mathrm{Mo}_{2} \mathrm{Ti}_{2} \mathrm{AlC}_{3}$. J. Appl. Phys. 2015, 118, 094304.

(37) Naguib, M.; Unocic, R. R.; Armstrong, B. L.; Nanda, J., Large-Scale Delamination of Multi-Layers Transition Metal Carbides and Carbonitrides "MXenes". Dalton Trans. 2015, 44, 9353-9358.

(38) Mashtalir, O.; Lukatskaya, M. R.; Kolesnikov, A. I.; Raymundo-Pinero, E.; Naguib, M.; Barsoum, M. W.; Gogotsi, Y., The Effect of Hydrazine Intercalation on the Structure and Capacitance of 2D Titanium Carbide (MXene). Nanoscale 2016, 8, 9128-9133.

(39) Fredrickson, K. D.; Anasori, B.; Seh, Z. W.; Gogotsi, Y.; Vojvodic, A., Effects of Applied Potential and Water Intercalation on the Surface Chemistry of $\mathrm{Ti}_{2} \mathrm{C}$ and $\mathrm{Mo}_{2} \mathrm{C}$ MXenes. J. Phys. Chem. C 2016, 120, 28432-28440.

(40) Sirisaksoontorn, W.; Adenuga, A. A.; Remcho, V. T.; Lerner, M. M., Preparation and Characterization of a Tetrabutylammonium Graphite Intercalation Compound. J. Am. Chem. Soc. 2011, 133, 12436-12438.

(41) Zhang, C. F.; Beidaghi, M.; Naguib, M.; Lukatskaya, M. R.; Zhao, M. Q.; Dyatkin, B.; Cook, K. M.; Kim, S. J.; Eng, B.; Xiao, X.; Long, D. H.; Qiao, W. M.; Dunn, B.; Gogotsi, Y., Synthesis and Charge Storage Properties of Hierarchical Niobium Pentoxide/Carbon/Niobium Carbide (MXene) Hybrid Materials. Chem. Mater. 2016, 28, 3937-3943.
(42) Naguib, M.; Saito, T.; Lai, S.; Rager, M. S.; Aytug, T.; Paranthaman, M. P.; Zhao, M. Q.; Gogotsi, Y., $\mathrm{Ti}_{3} \mathrm{C}_{2} \mathrm{~T}_{\mathrm{x}}$ (MXene)Polyacrylamide Nanocomposite Films. RSC $A d v$. 2016, 6, 7206972073.

(43) Xie, Y.; Naguib, M.; Mochalin, V. N.; Barsoum, M. W.; Gogotsi, Y.; Yu, X. Q.; Nam, K. W.; Yang, X. Q.; Kolesnikov, A. I.; Kent, P. R. C., Role of Surface Structure on Li-Ion Energy Storage Capacity of Two-Dimensional Transition-Metal Carbides. J. Am. Chem. Soc. 2014, 136, 6385-6394.

(44) Li, L. H., Lattice Dynamics and Electronic Structures of $\mathrm{Ti}_{3} \mathrm{C}_{2} \mathrm{O}_{2}$ and $\mathrm{Mo}_{2} \mathrm{TiC}_{2} \mathrm{O}_{2}$ (MXenes): The Effect of Mo Substitution. Comput. Mater. Sci. 2016, 124, 8-14.

(45) Li, Y. F.; Ding, Y. C.; Xiao, B.; Cheng, Y. H., Anisotropic Electrical and Lattice Transport Properties of Ordered Quaternary Phases $\mathrm{Cr}_{2} \mathrm{TiAlC}_{2}$ and $\mathrm{Mo}_{2} \mathrm{TiAlC}_{2}$ : A First Principles Study. Phys. Lett. A 2016, 380, 3748-3755.

(46) Oh, J. Y.; Lee, J. H.; Han, S. W.; Chae, S. S.; Bae, E. J.; Kang, Y. H.; Choi, W. J.; Cho, S. Y.; Lee, J. O.; Baik, H. K.; Il Lee, T., Chemically Exfoliated Transition Metal Dichalcogenide NanosheetBased Wearable Thermoelectric Generators. Energy Environ. Sci. 2016, 9, 1696-1705.

(47) Kuroki, K.; Arita, R., "Pudding Mold" Band Drives Large Thermopower in $\mathrm{Na}_{\mathrm{x}} \mathrm{CoO}_{2}$. J. Phys. Soc. Jpn. 2007, 76, 083707.

(48) Mori, K.; Sakakibara, H.; Usui, H.; Kuroki, K., Ideal Band Shape in the Potential Thermoelectric Material $\mathrm{CuAlO}_{2}$ : Comparison to $\mathrm{Na}_{\mathrm{x}} \mathrm{CoO}_{2}$. Phys. Rev. B 2013, 88, 075141.

(49) Usui, H.; Suzuki, K.; Kuroki, K.; Nakano, S.; Kudo, K.; Nohara, M., Large Seebeck Effect in Electron-Doped FeAs 2 Driven by A Quasi-One-Dimensional Pudding-Mold-Type Band. Phys. Rev. B 2013, 88, 075140 .

(50) Si, C.; You, J.; Shi, W.; Zhou, J.; Sun, Z., Quantum Spin Hall Phase in $\mathrm{Mo}_{2} \mathrm{M}_{2} \mathrm{C}_{3} \mathrm{O}_{2}(\mathrm{M}=\mathrm{Ti}, \mathrm{Zr}$, Hf) MXenes. J. Mater. Chem. C 2016, 4, 11524-11529.

(51) Lu, H.; Burke, P. G.; Gossard, A. C.; Zeng, G.; Ramu, A. T.; Bahk, J. H.; Bowers, J. E., Semimetal/Semiconductor Nanocomposites for Thermoelectrics. Adv. Mater. 2011, 23, 2377-2383.

(52) Chen, Y.; He, M.; Liu, B.; Bazan, G. C.; Zhou, J.; Liang, Z., Bendable n-Type Metallic Nanocomposites with Large Thermoelectric Power Factor. Adv. Mater. 2017, 29, 1604752. 
Insert Table of Contents artwork here
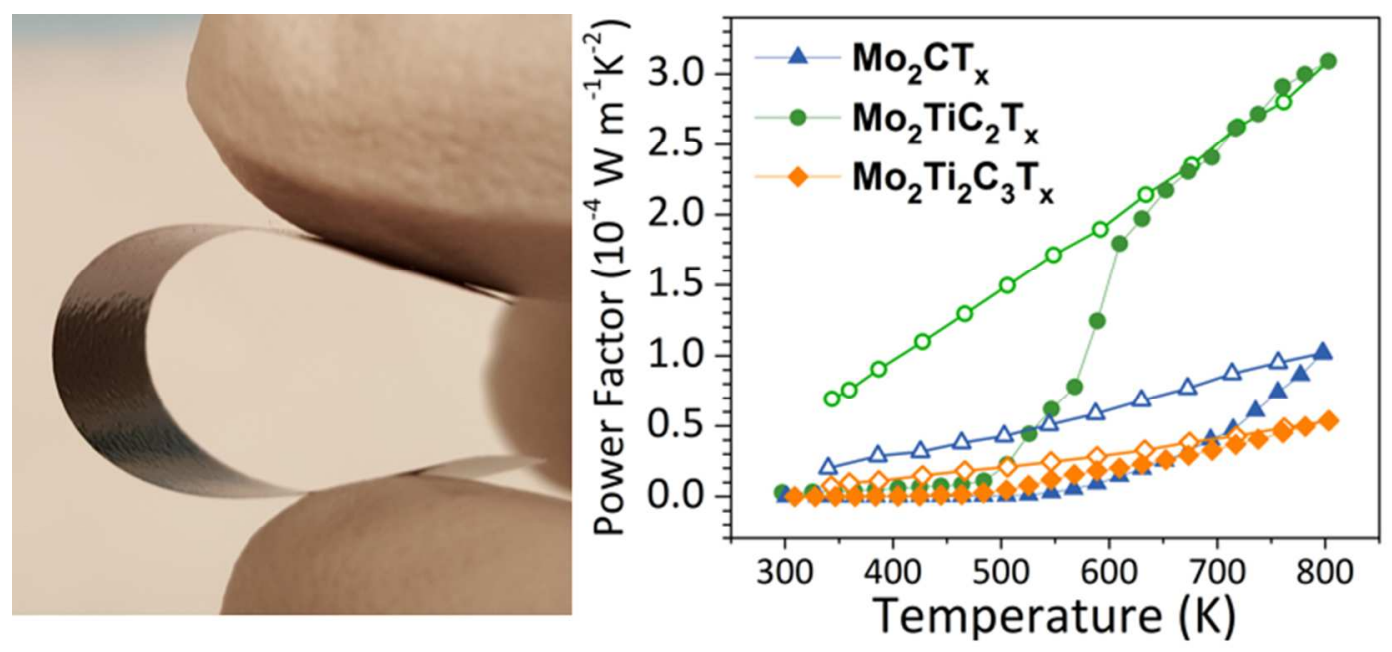

Mo OTi OC
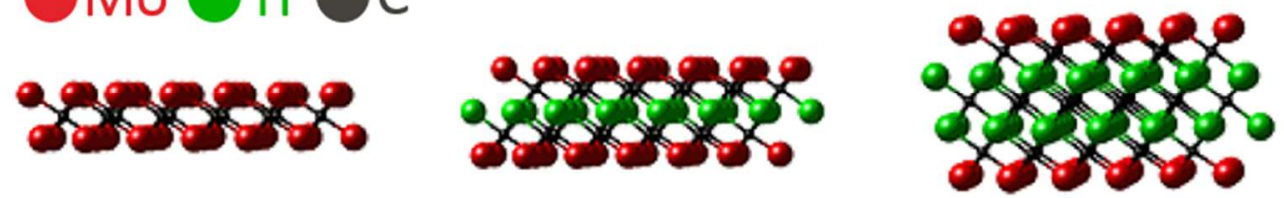

$\mathrm{Mo}_{2} \mathrm{C}$

$\mathrm{Mo}_{2} \mathrm{TiC}_{2}$

$\mathrm{Mo}_{2} \mathrm{Ti}_{2} \mathrm{C}_{3}$ 